\title{
Coupling of Monte Carlo Sputter Simulation and Feature-Scale Profile Simulation and Application to the Simulation of Back Etching of an Intermetal Dielectric
}

\author{
E. Baer, D. Kunder, J. Lorenz \\ Fraunhofer Institute for Integrated Systems and \\ Device Technology IISB \\ Schottkystrasse 10, 91058 Erlangen, Germany \\ eberhard.baer@iisb.fraunhofer.de
}

\begin{abstract}
We demonstrate the coupling of Monte Carlo sputter simulation with feature-scale simulation of profile evolution during sputter etching. With the Monte Carlo sputter simulation, the dependence of the sputter yield on the angle of incidence and on the energy of ions impinging onto the surface is determined. The yield curves obtained thereby are fed into a feature-scale etching profile simulator which predicts the local etch rates based on these sputter yield curves and on ion fluxes which are calculated for a substrate placed in a sputter reactor. For validating the simulations, a process sequence consisting of deposition and back etching (in an argon plasma) of silicon oxide between metal lines has been studied. Assuming an ion energy of $250 \mathrm{eV}$ allows us to consistently reproduce profiles observed experimentally.
\end{abstract}

\section{INTRODUCTION}

The evolution of microstructures during physical or chemical etching processes can be predicted by numerical feature scale simulation. As input to the simulation, information about the incoming fluxes of neutral and charged particles is needed as well as knowledge about the interaction between the particles and the surface of the feature to be etched.

In this paper we present the extension of an approach which has been previously demonstrated for ion beam etching [1] to a full area physical sputter etching process. We demonstrate the coupling between Monte Carlo simulation for determining the sputter yield and feature-scale simulation taking these yield data as input for predicting surface evolution. Application to back etching of an intermetal dielectric (silicon oxide) is shown, including comparison with experimental data.

This work was supported by the Fraunhofer Internal Programs under Grant No. MAVO 817759 (HIESPANA).

\author{
M. Sekowski \\ Chair of Electron Devices, University of Erlangen-Nuremberg \\ Cauerstrasse 6, 91058 Erlangen, Germany \\ U. Paschen
Fraunhofer Institute for Microelectronic
Systems and Circuits IMS
Finkenstrasse 61, 47057 Duisburg, Germany \\ U. Paschen
Fraunhofer Institute for Microelectronic
Systems and Circuits IMS
Finkenstrasse 61, 47057 Duisburg, Germany \\ U. Paschen
Fraunhofer Institute for Microelectronic
Systems and Circuits IMS
Finkenstrasse 61, 47057 Duisburg, Germany \\ U. Paschen
Fraunhofer Institute for Microelectronic
Systems and Circuits IMS
Finkenstrasse 61, 47057 Duisburg, Germany
}

\section{Monte CARLo Simulation of Physical SPUTTERING}

Information about the sputtering yield was obtained from MC_SIM [2], a Monte Carlo ion implantation program, which calculates the trajectories of primary and recoiled atoms on the basis of the binary collision approximation [3]. The sputtering yield depends on the model for electronic stopping. As reported [1], two models for electronic stopping were adjusted to the case of gallium ions impinging upon a silicon target with an energy of $30 \mathrm{keV}$. For this combination of ion and target, we have obtained good agreement between simulation and experiment for different angles of incidence.

For the sputtering of silicon dioxide with argon ions, which is the system studied in this paper, we applied a target density of $2.63 \mathrm{~g} / \mathrm{cm}^{3}$ and the calibration for the electronic stopping model mentioned above. For an ion energy of 600 $\mathrm{eV}$, the sputtering yield is in good agreement between simulation and experiment [4]. The sputtering yields were calculated for different angles of incidence and for different ion energies (Figure 1).

\section{FEATURE-SCAle Simulation OF Profile Evolution}

\section{A. Physical Model for Feature Evolution during Sputter Etching}

For feature-scale simulation of sputter etching, the local etch rate is determined by the local flux of ions and the sputter yield which in our case is delivered by MC_SIM.

For the process considered, the material removal is exclusively due to the physical process of sputtering. No 
chemical reactions are involved as it would be the case for the process of reactive ion etching (RIE) where material removal to a large extent is due to a combined mechanism involving physical as well as chemical processes. During a RIE process, e.g., reactive molecules can react with the substrate forming a non-volatile reaction product. Under bombardment of energetic ions this product can desorb. Therefore, the etching mechanism is based on combined physical and chemical effects.

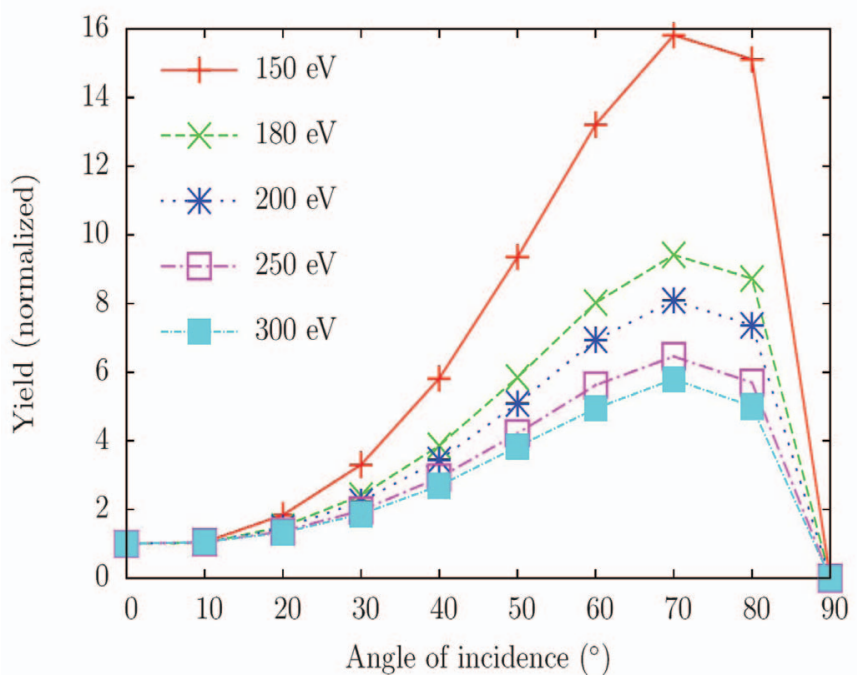

Figure 1. Angular characteristics of the silicon oxide sputter yield for Ar ions with different energies, simulated with the Monte Carlo sputter simulator MC SIM. The yield shown is a relative one, i.e. the ratio of the yield and the yield for an angle of incidence of 0 .

In contrast, in the work described in this paper, the material removal is due to energy transfer of the incoming ions to the atoms of the substrate. If the energy transferred is high enough, the substrate atoms are ejected leading to material removal, called sputter etching. The details of the physical interaction mechanisms are modeled by the program MC SIM as described in Section II. The simulations with MC_SIM are not carried out in-situ during feature-scale simulation but they are used to generate data describing the sputter yield and its dependence on the angle of incidence and on the energy of the ions. These data tables are fed into the feature scale simulation for predicting the interaction of ions with the surface needed by the feature scale sputter etching model.

The local etch rate $E R$ for a position on the feature surface is obtained by integrating the product of the local ion flux $I$ (ions per surface area and time) and the sputter yield $Y$. The integration is carried out over the solid angle $\Omega_{\text {free }}$ with free view to the reactor for the position under consideration:

$$
E R \propto \int_{\Omega_{\text {free }}} d \Omega I\left(\vartheta_{\text {glob }}, \varphi_{g l o b}\right) Y\left(\vartheta_{l o c}\right)
$$

The local ion flux $I$ depends on the global coordinates, i.e. the coordinates related to the reactor geometry. The sputter yield $Y$ depends on the local coordinates, i.e. the orientation of the local surface normal with respect to the direction of the incoming ions. Without considering effects due to crystal orientation, the latter leads to a dependence on $\vartheta_{l o c}$ only. In order to determine ER, the etch rate in planar sections (without any $2 \mathrm{D}$ or $3 \mathrm{D}$ structure) $E R_{\text {planar }}$ needs to be considered and is given by:

$$
E R_{\text {planar }} \propto \int_{\text {Half unit sphere }} d \Omega I\left(\vartheta_{\text {glob }}, \varphi_{\text {glob }}\right) Y\left(\vartheta_{l o c}\right)
$$

The only difference of (2) in comparison to (1) is the solid angle of integration. For the planar sections no shadowing of the ion flux by the structure takes place. Therefore the integration is carried out over the half unit sphere corresponding to free view to the reactor and a solid angle of $2 \pi$.

For the simulations shown in this work, we do not predict the value of the etch rate itself which depends e.g. on the reactor settings, but we are interested in the relative variation of the etch rate along the feature surface. When the thickness etched in planar sections $E T_{\text {planar }}$ is prescribed, as it is the case for the application shown below, it is even not necessary to know the etch rate. In this case, the locally etched thickness $E T$ is the quantity of interest which is obtained by:

$$
E T=E T_{\text {planar }} \cdot E R / E R_{\text {planar }}
$$

There are quantities used in (1) which change during the etching process due to the change of the surface profile, namely the solid angle of free view $\Omega_{\text {free }}$ and the surface normal which impacts on the value of $\vartheta_{\text {loc }}$ which is relevant for determination of the local sputter yield. Therefore, the simulation has to be carried out in a number of steps (100 steps have been used for the example shown below) to make sure that for a "tracked" position on the surface the ratio $E R / E R_{\text {planar }}$ does not change significantly within one simulation time step. Such a simulation time step corresponds to part of the total etch thickness and for each simulation time step the integration according to (1) is performed again. The number of time steps for which sufficient accuracy is achieved has been determined empirically, i.e. the number of steps has been increased until no further changes of the simulated geometry have been observed. Though it might be desirable to predict the number of steps a-priori, based e.g. on analytical considerations, this might be of limited applicability for general structures, or for systems with strongly varying local rates as it is the case for the example shown below.

The angular distribution of ions can be provided by equipment simulation or measurements and determines the quantity $I$ referred to in (2) and (3).

\section{B. Etching Model Implementation}

The representation of the geometry in our simulator is achieved by discretizing the surface using triangular facets. This means that the simulator in principle allows full 3D treatment of structures. However, for the work shown in this paper, the simulator has been used in a $2 \mathrm{D}$ mode, due to the experimental data which are related to structures defined by long metal lines which can be well treated by a 2D simulation. 
The determination of the local etch rate according to (1) and (2) can be performed in different ways. To this end, our simulation program has a Monte Carlo module as well as a numerical flux integration module. Within the Monte Carlo module, pseudo-particles are launched according to the angular distribution of ions and their point of impact on the surface is determined assuming ballistic transport. According to the angle of impact, the contribution to the etch rate is predicted and collected triangle-wise. The numerical flux integration module performs a discrete scanning (with respect to the triangle midpoint position of the surface grid) of spatial directions and sums up the corresponding contributions to etching depending on the flux intensity and the angle with the surface normal (both for the direction under consideration). For both approaches, determination of surface shadowing needs to be carried out. In terms of calculation times, the numerical flux integration module outperforms the Monte Carlo module. However, the Monte Carlo module allows a straightforward extension towards further etching (or redeposition) mechanisms which might be more difficult to implement in the numerical flux integration module. For the work shown in this paper, the modeling level available in the flux integration module is sufficient for the effects we want to study. Therefore, this module has been chosen for the etching simulations shown below.

\section{Modeling of the Oxide Deposition Process}

The geometry at the beginning of the etching process is generated by plasma-enhanced chemical vapor deposition (PECVD) of silicon oxide on patterned metal lines. As the modeling of the deposition process is not in the focus of the paper, the approach is only briefly described in the following. For the simulation of PECVD, a model developed by Li and coworkers [5] has been adopted. In the model, deposition is attributed to the oxidation of precursor molecules or fragments adsorbed on the surface. The oxidation can result either from impinging oxygen radicals (neutrals) or oxygen ions. The neutrals are assumed to have an isotropic angular distribution and a sticking coefficient below unity. For the ions, a unity sticking coefficient and an angular distribution described by a Gaussian distribution are presumed. The local rate consists of a neutral-induced rate and an ion-induced rate. The parameters required by the model are the neutral contribution to the overall growth rate in planar ("1D") regions, the sticking coefficient of the neutrals, and the standard deviation of the Gaussian distribution that describes the angular distribution of the ion flux. The model implementation in the deposition simulator DEP3D [6] has been used for the simulations shown in this paper.

\section{APPLICATION EXAMPLE}

We show results of a process sequence of oxide deposition using PECVD followed by physical sputter etching of the oxide layer. The basic goal of this sequence is to open a void potentially created during the non-conformal deposition process to make the structure suitable for further processing. The process is carried out as follows: First, silicon dioxide is deposited on top of the metal lines using PECVD (1D deposited thickness [i.e. thickness in planar sections] is 450 $\mathrm{nm})$. Next, back etching is carried out by physical sputter etching (1D etched thickness is $100 \mathrm{~nm}$ ) in an argon plasma. The experimental data (cross section) are shown in Figure 2, consisting of profiles for two trenches with different aspect ratios.

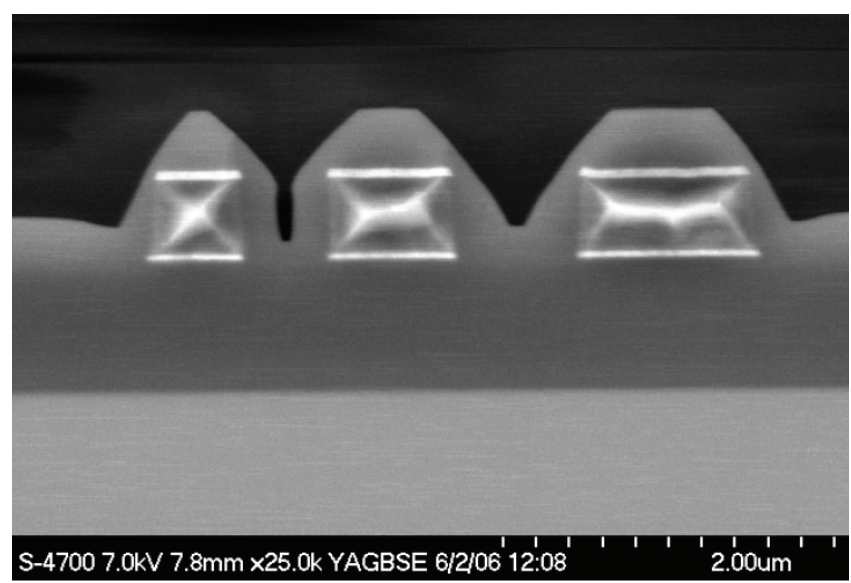

Figure 2. Experimental profiles of oxide on metal lines after plasmaenhanced chemical vapor deposition followed by physical sputter etching.

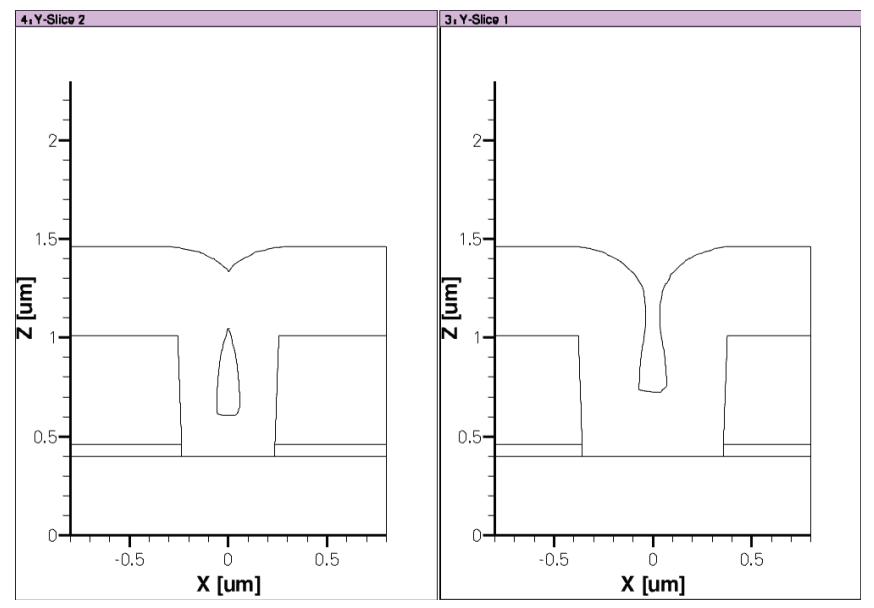

Figure 3. Simulated profiles of deposited oxide on trenches with different aspect ratios. A void has been formed for the trench shown on the left. This void is opened again during the subsequent etching process.

To obtain the correct PECVD model parameters for the process studied in the experiment, the profile of the wider trench (right trench in Figure 2) after etching has been used. With respect to the profile after etching, $100 \mathrm{~nm}$ of oxide thickness have been "added" on both the trench bottom and the trench top to allow calculation of the bottom coverage directly after PECVD. Here, we have assumed that due to the directionality of sputter etching the removed thickness is the same at the bottom and at the top of the trench structure, namely $100 \mathrm{~nm}$. For a relative contribution of the neutrals to the growth rate in $1 \mathrm{D}$ regions of 1 , a neutral sticking coefficient of 0.18 and a standard deviation of the Gaussian distribution describing the angular distribution of ions of 0.05 the simulations predict the correct value of the bottom coverage. The simulated profiles after deposition are shown in 
Figure 3. As for the left trench a void is formed, the amount of oxide thickness removed during etching is less than $100 \mathrm{~nm}$, because the bottom of the trench is open only after the void reopens again during the etching process. Therefore, it is not possible to use the profile of the left trench for the PECVD model calibration described above.

For the etching simulation, the angular distribution of the Ar ions was approximated by a Gaussian function with a standard deviation $\sigma$ of 0.02 . Such a value is typically obtained for equipment setups used for physical sputter etching processes [7]. However, it has been shown that for the etching process considered here, the influence of $\sigma$ is not very pronounced. This is due to the fact that the most important effect observed in the profiles is the faceting which is predominantly governed by the angular dependence of the sputter yield.

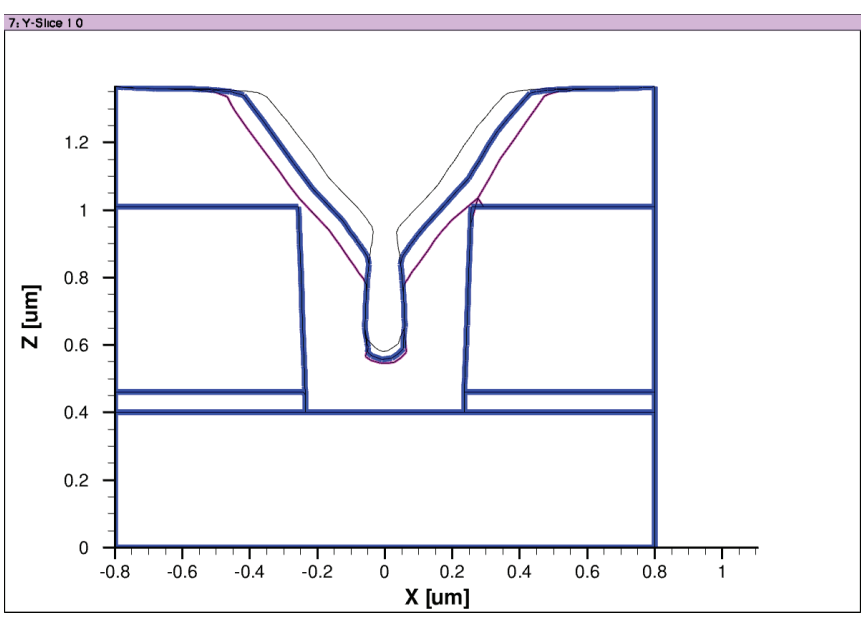

Figure 4. Simulated profiles after sputter etching for different energies of the Ar ions (upper curve: $250 \mathrm{eV}$, middle curve: $200 \mathrm{eV}$, lower curve: $180 \mathrm{eV})$.

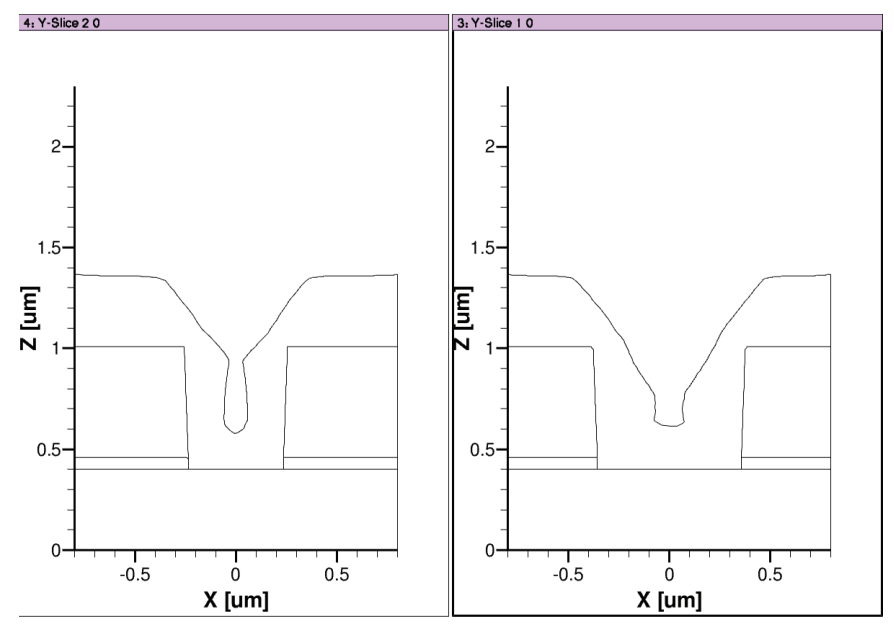

Figure 5. Simulation results for the profiles after back etching of the deposited oxide. For both trenches, good agreement with the experimental data shown in Figure 2 is achieved.
The simulation of the physical sputter back etching of the oxide layer has been carried out for different ion energies, using the respective yield curves as shown in Figure 1. The results are shown in Figure 4 for energies of $180 \mathrm{eV}, 200 \mathrm{eV}$, and $250 \mathrm{eV}$. For an ion energy of $250 \mathrm{eV}$ good agreement with the experimentally observed profile is observed. This is not only the case for the left trench with the higher aspect ratio but also the profile for the right trench with the smaller aspect ratio is well reproduced (Figure 5). The remaining differences, particularly for the right trench can be attributed to the fact, that in the model used the redeposition of sputtered metal is not considered but may affect the profile in reality.

\section{CONCLUSIONS}

We have demonstrated the coupling of a Monte Carlo sputter simulator (MC_SIM) with feature-scale simulation of profile evolution during sputter etching. The Monte Carlo simulator MC_SIM has been used to determine the dependence of the sputter yield on the angle of incidence of the ions. The simulations have been carried out for various energies of the incoming ions. The yield curves obtained by MC_SIM have been fed into a feature-scale etching profile simulator. In this simulator, the local ion fluxes are determined and are used to calculate local etch rates.

As an application example, a process sequence used for filling the gaps between metal lines with silicon oxide has been studied. The process sequence consists of deposition of silicon oxide followed by sputter back etching of the oxide. Assuming an ion energy of $250 \mathrm{eV}$ allows us to predict the profiles of the oxide layer after back etching in two trenches with different aspect ratios. The simulation data show good agreement with experimental measurements.

\section{REFERENCES}

[1] D. Kunder, E. Baer, M. Sekowski, P. Pichler, M. Rommel, "Simulation of focused ion beam etching by coupling a topography simulator and a Monte Carlo sputtering yield simulator," Microelectr. Eng., vol. 87, pp. 1597-1599, May-August 2010.

[2] M. Ullrich, A. Burenkov, and H. Ryssel, "Ion sputtering at grazing incidence for SIMS-analysis,” Nucl. Instr. Meth. Phys. Research B, vol. 228, pp. 373-377, January 2005.

[3] J.P. Biersack and W. Eckstein, "Sputtering studies with the Monte Carlo program TRIM.SP," Appl. Phys. A (Materials Science \& Processing), vol. 34, pp. 73-94, June 1984.

[4] T.M. Mayer, R.A. Barker, and N.J. Whitman, "Investigation of plasma etching mechanisms using beams of reactive gas ions," J. Vac. Sci Technol., vol. 18, pp. 349-352, March 1981.

[5] J. Li, J. P. McVittie, J. Ferziger, and K.C. Saraswat, "Optimization of intermetal dielectric deposition module using simulation,” J. Vac. Sci. Technol., vol. B 13, pp. 1867-1874, July-August 1995.

[6] Deposition simulator DEP3D, release 0.6, Fraunhofer IISB, Erlangen, 2008.

[7] M. Kratzer, R.P. Brinkmann, W. Sabisch, H. Schmidt, "Hybrid model for the calculation of ion distribution functions behind a direct current or radio frequency driven plasma boundary sheath," J. Appl. Phys., vol. 90, pp. 2169-2179, September 2001 